Scientific Paper

\title{
Automatic validation of the gantry tilt in a computed tomography scanner using a head polymethyl methacrylate phantom
}

\author{
Rakhma NOVILIAWATI ${ }^{\mathrm{a}}$, Choirul ANAM ${ }^{\mathrm{a},{ }^{*},}$, Heri SUTANTO ${ }^{\mathrm{a}}$, Geoff DOUGHERTY ${ }^{\mathrm{b}}$, Muhammad Ridha MAK'RUF ${ }^{\mathrm{c}}$ \\ ${ }^{a}$ Department of Physics, Faculty of Sciences and Mathematics, Diponegoro University, Jl.Prof. Soedarto SH, Tembalang, \\ Semarang 50275, Central Java, Indonesia \\ ${ }^{b}$ Department of Applied Physics and Medical Imaging, California State University Channel Islands, Camarillo, CA 93012, \\ USA \\ ${ }^{c}$ Department of Electromedical Engineering, Poltekkes Kemenkes, Surabaya, East Java, Indonesia \\ *E-mail address: anam@fisika.fsm.undip.ac.id
}

\begin{abstract}
The purpose of this study was to develop an automatic method for validating the computed tomography gantry tilt. A head polymethyl methacrylate phantom with a diameter of $16 \mathrm{~cm}$ was used. Gantry tilt angles were measured both manually and automatically. Manual measurements were performed by measuring the length of the anteroposterior and lateral diameters from acquired images using electronic calipers. Automatic measurements consisted of a number of steps: phantom segmentation, determination of the center of the phantom, measurement of the anteroposterior and lateral diameters, and computation of the gantry tilt angle. The method was implemented on the gantry angles from $0^{\circ}$ to $15^{\circ}$. The proposed method of measuring gantry angles produced accurate gantry tilt angles. The differences with the angles displayed on the gantry were less than $1^{\circ}$. The results of the automatic method were the same as those of the manual method $\left(\mathrm{R}^{2}>0.98\right)$.
\end{abstract}

Key words: computed tomography; gantry tilt; polymethyl methacrylate phantom; automated method; quality control.

\section{Introduction}

The computed tomography (CT) scanner is used primarily for medical purposes. ${ }^{1}$ It delivers more diagnostic information compared to conventional X-ray techniques. ${ }^{2}$ The speed of acquisition of CT scanners is an advantage that makes it so widely used in clinical examinations. The first CT scanner took approximately $300 \mathrm{~s}$ to acquire an image of a single slice, ${ }^{3}$ but now hundreds of slices can be obtained just in one rotation of the X-ray tube in less than a second.

The CT scanner employs a relatively high dose of ionizing radiation so that the risk to the patient can be considerable. Dosimetry in CT scanners has specific methods and criteria because of the specificity of the CT scanner modality. ${ }^{4} \mathrm{~A}$ diagnostic image quality is crucial in establishing the diagnosis, and it must be obtained with the lowest possible dose. This requires dose optimization. ${ }^{5}$

A CT procedure that is often performed is head examination. Head CT examinations in clinical practices often lead to the eye lens being exposed to the primary radiation. ${ }^{3,6}$ The dose threshold determined by the International Commission on Radiological Protection (ICRP) for the eye lens is $500 \mathrm{mGy}$, and eye dose limit for cataract formation is $2000 \mathrm{mGy}{ }^{6,7}$ Generally, the dose for head CT examination is approximately $50 \mathrm{mGy} .^{5-11}$
For head CT examinations where the organ of interest is located behind the eye lens, the CT gantry is often tilted so that primary radiation does not directly impinge the eye lens. ${ }^{10}$ Figure 1 shows that the eye lens is exposed to primary radiation when the orbitomeatal line is used as the baseline. However, when the radiation is parallel to the supraorbitomeatal line, the eye lens is only exposed to the scattered radiation. $^{7}$

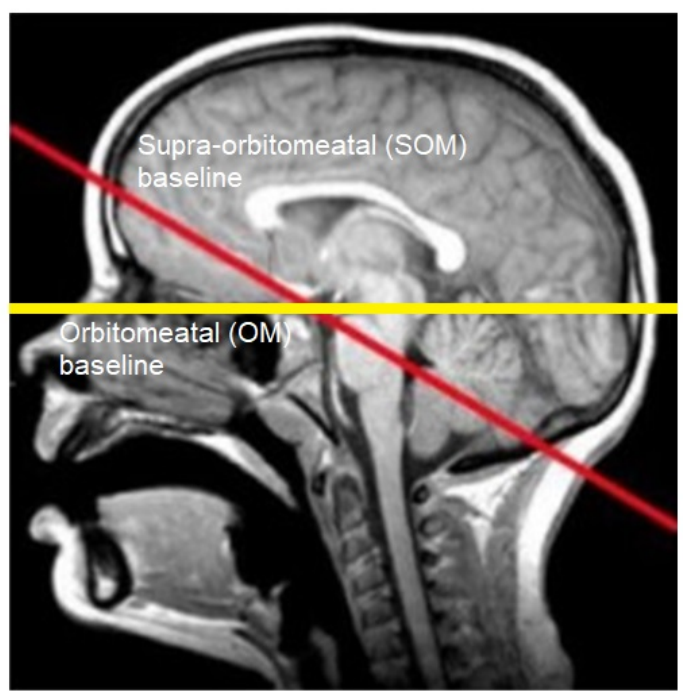

Figure 1. Supra-orbitomeatal (SOM) and orbitomeatal (OM) baselines in head computed tomography examination. 
The amount of scattered radiation impinging the eye lens depends on the distance between the primary radiation and the eye lens. ${ }^{10}$ A previous study reported that gantry tilting with the eye lens outside the primary radiation could reduce radiation exposure to the eye lens by up to $75 \% .^{11}$

The gantry tilt is the angle between the vertical plane to the isocenter and the CT gantry. ${ }^{12,13}$ It is often performed for clinical purposes and is set manually by the operator. It can be performed from angles of $-30^{\circ}$ to $+30^{\circ}$ in $0.5^{\circ}$ increments according to the specifications of the CT scanner and specific examination. $^{14}$

The gantry tilt angle accuracy should be periodically evaluated $^{15}$ so that the magnitude of the gantry tilt degree indicated on the gantry display matches the actual gantry tilt degree. ${ }^{5,8}$ The method for validating the gantry tilt is generally with the use of an imaging plate in computed radiography (CR) or a protractor (film radiography), which is placed perpendicular to the table in the sagittal plane. ${ }^{16}$ The plate or film is scanned in the axial mode throughout the angles of the gantry tilt range. The angle is measured from the image acquired by CR or film. ${ }^{17}$ Three axial exposures are generally made with the same plate or film, at angles $\Theta_{-}^{-}$, angle $\Theta_{+}$, and $0^{\circ}$. Three scanning tracks will be seen in the plate or film. The angles of $\Theta$ - and $\Theta+$ at gantry tilt relative to the tilt at $0^{\circ}$ must be the same as the tilt angle displayed on the gantry. ${ }^{18}$ The acceptable limit of the difference of gantry tilt is within $3^{\circ} .{ }^{18}$

An alternative measurement of gantry tilt accuracy can be performed using a cylindrical water phantom or the polymethyl methacrylate (PMMA) phantom generally used for dosimetry. Two scans of the phantom are performed. The first scan is carried out with a gantry angle of $0^{\circ}$, and the second scan is carried out at a certain angle to be evaluated. The accuracy of the gantry tilt is ascertained by comparing the length of the diameters in the anteroposterior (AP) direction for the two scans. ${ }^{16}$ Evaluation with double scanning is undoubtedly less efficient and subjective when evaluated manually.

The current study was aimed at proposing a method to validate the accuracy of the gantry tilt with only one scan using a readily available phantom. In addition, we aimed to develop an automated system for calculating the gantry tilt using the software so that measurements are faster and more efficient.

\section{Methods}

\section{CT scanner and phantom}

We used a head PMMA phantom with a diameter of $16 \mathrm{~cm}$ and length of $15 \mathrm{~cm}$, as commonly used to measure the output dose of CT in terms of $\mathrm{CTDI}_{\mathrm{vol}}$. Gantry tilts varied at angles of $0^{\circ}$, $5^{\circ}, 10^{\circ}$, and $15^{\circ}$. Measurements were carried out at two slice thicknesses, i.e. 1.5 and $5 \mathrm{~mm}$. The phantom was scanned by the Siemens Somatom 16 CT scanner in the axial mode with parameters of $130 \mathrm{kVp}$ and $240 \mathrm{~mA}$. The image was reconstructed by filtered back-projection with Syngo CT2009E
(Siemens) with the H31s convolution kernel. Figure 2 shows the head PMMA phantom in the CT scanner table.

\section{Calculation of gantry angle}

In this study, gantry tilt angles were measured both manually and automatically. Manual gantry tilt angle measurements were performed by measuring the length of the AP and lateral (LAT) diameters from the acquired images using the electronic calipers available in the CT computer console (Figure 3). The gantry angles were computed using Equation 1.

$\theta=\cos ^{-1}\left(\frac{L A T}{A P}\right)$

Eq. 1

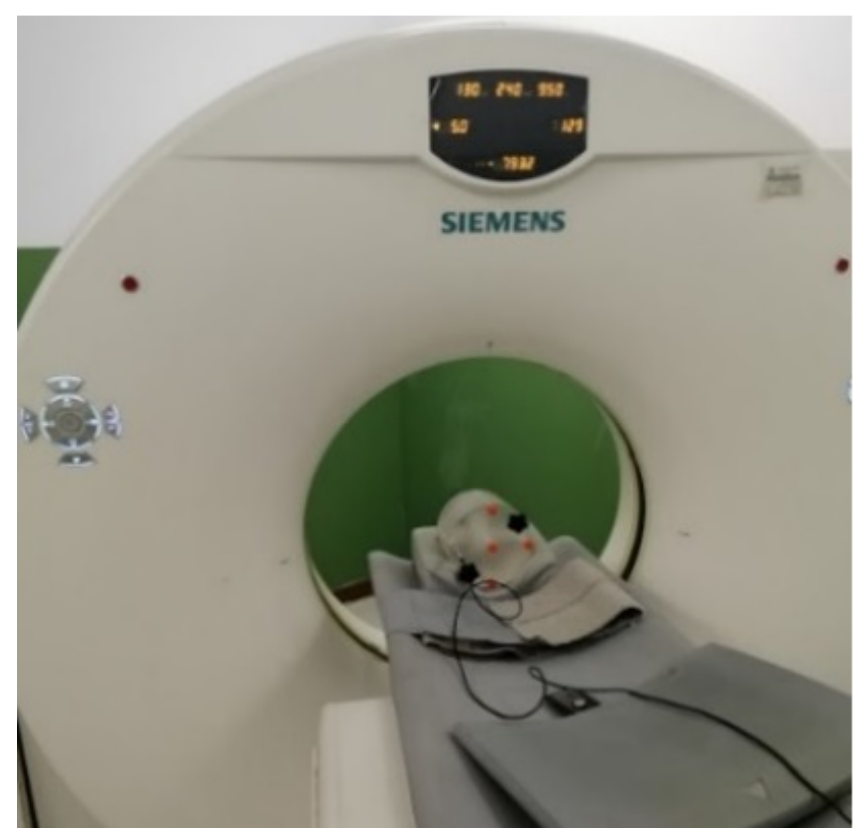

Figure 2. Photograph of a polymethyl methacrylate phantom in the computed tomography scanner.

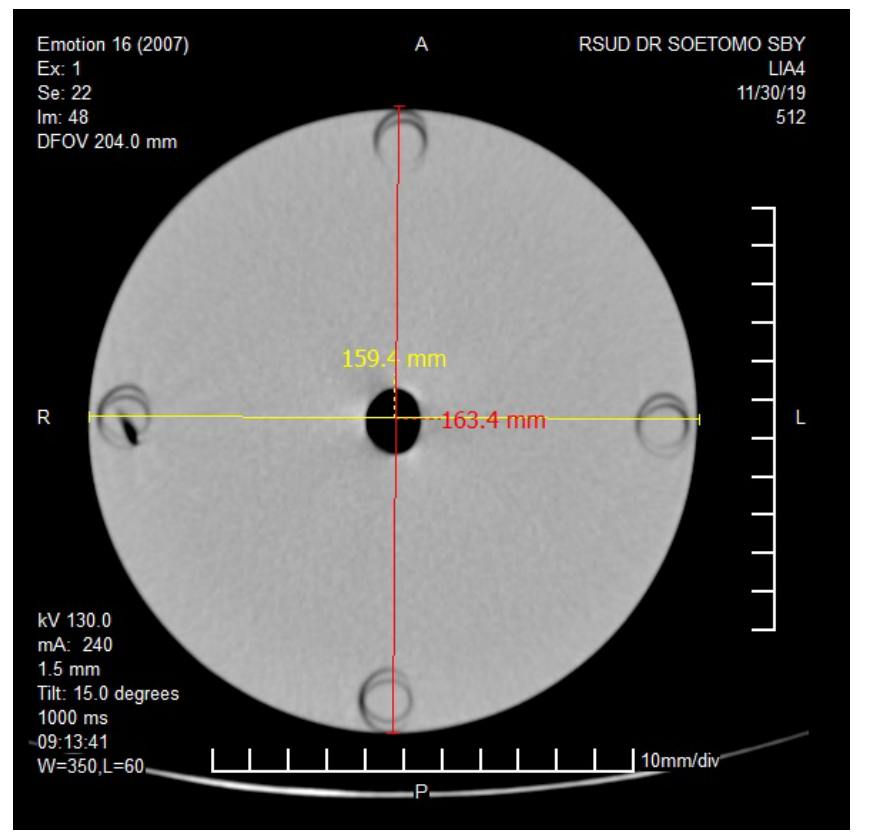

Figure 3. Manual AP and LAT diameter for measuring gantry tilt angle using an electronic caliper. 
The automatic gantry tilt angle calculation consisted of several steps (Figure 4). The first was to automatically segment phantom images (Figure 4(a)) using an algorithm introduced previously by Anam et al. ${ }^{19}$. This segmentation was proven to be accurate with an error of less than $0.5 \% .^{20,21}$ The second was to automatically determine the center of the phantom image using the centroid equation. Next, the lengths of the LAT and AP diameters were automatically determined, and gantry angle was calculated based on a comparison of LAT and AP diameters.

The automatic image segmentation was a multi-step process. We converted the image from grayscale to binary image using an image threshold of -200 HU and determined the edges of all objects within the image. After labelling the objects, we calculated their areas. The widest object was considered the border of the phantom. Finally, we filled the phantom within its boundary with a value of 1 . An example of the segmentation result is shown in Figure 4(b).

The center of the phantom was determined by the centroid Equation 2.

$\left(x_{c}, y_{c}\right)=\frac{1}{N} \sum_{i=1}^{N}\left(x_{i}, y_{i}\right)$

where $x_{i}$ and $y_{i}$ are the positions of the pixels in the segmented phantom image. An example of the phantom center result is shown in Figure 4(c). The center of the phantom is indicated by a red dot.

Subsequently, diameters of AP and LAT were automatically determined. The AP diameter was defined as the sum of all pixels $(f)$ from $j=1$ to $\mathrm{n}$ in the $\mathrm{x}_{\mathrm{c}}$ position, and the LAT diameter was determined as the sum of all the pixels from $i=1$ to $\mathrm{n}$ in the $\mathrm{y}_{\mathrm{c}}$ position. Each $f$ was multiplied by $d$, which is the distance between pixels in the image taken from the DICOM header.

$$
\begin{aligned}
& A P=d \times \sum_{j=1}^{n} f_{x c, j} \\
& L A T=d \times \sum_{i=1}^{n} f_{i, y c}
\end{aligned}
$$

An example of an image with lines indicating the AP and LAT diameters is shown in Figure 4(d). Vertical lines indicate the AP diameter, and horizontal lines indicate the LAT diameter.

The gantry angle was automatically calculated using Equation 1. ${ }^{16}$ However, for a gantry angle of $0^{\circ}$, the AP diameter might be shorter than the LAT diameter. In this case, the gantry angle was calculated using Equation 5.

$\theta=-\cos ^{-1}\left(\frac{A P}{L A T}\right)$

The results must have errors within $3^{\circ}$ from the set gantry tilt or displayed value in the gantry. ${ }^{19}$ The graphical user interface of the gantry tilt calculation is shown in Figure 5. The AP and LAT diameters and the gantry tilt were measured 20 slices of every gantry angle, and mean and standard deviations were computed.
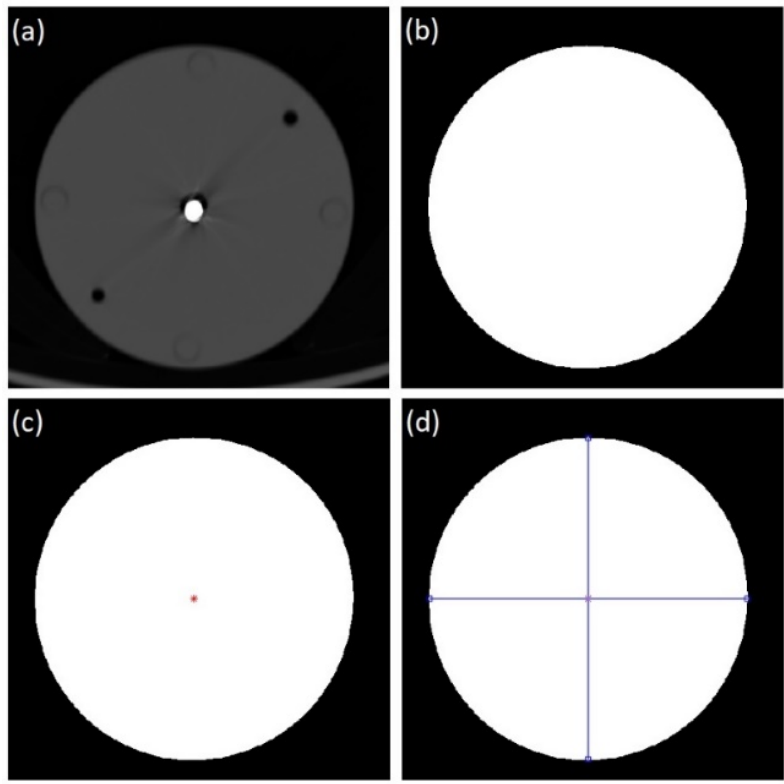

Figure 4. Steps of the segmentation process, (a) phantom image, (b) phantom binary image resulting from auto-segmentation, (c) image with a center phantom (as indicated by a red dot), and (d) phantom image with lines indicating diameters in lateral and anteroposterior directions to measure the gantry tilt.

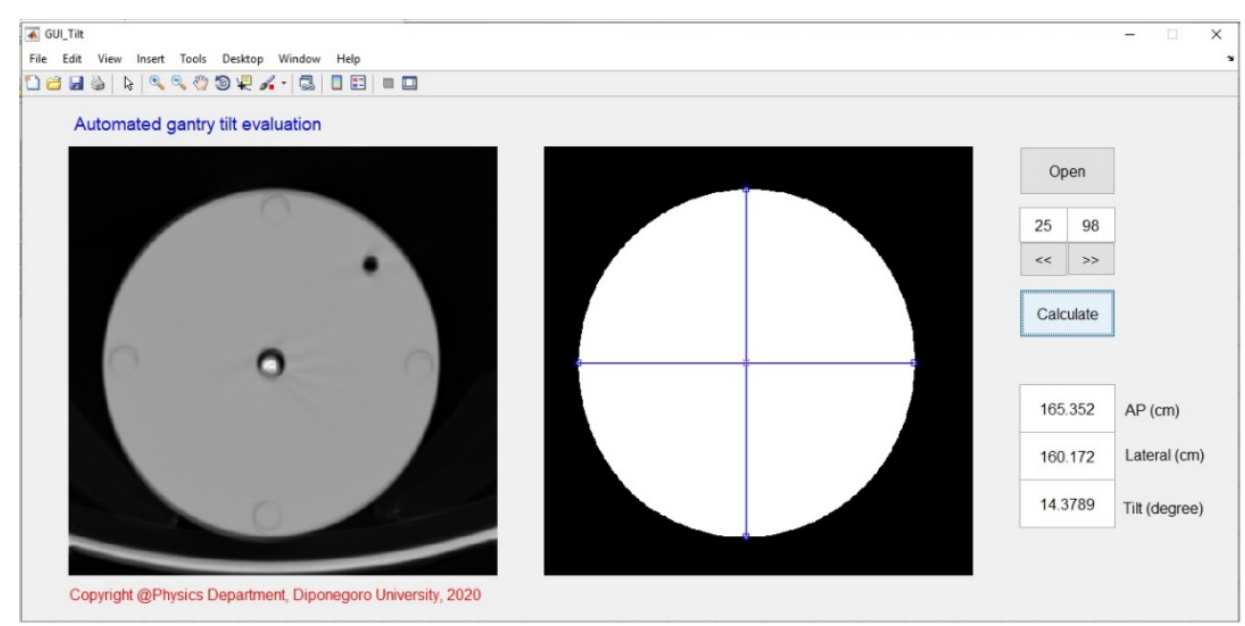

Figure 5. Screenshot of graphical user interface (GUI) for automatic gantry tilt calculation. An example of an original phantom image (left) and segmented phantom image with lines indicating the anteroposterior and lateral diameters. The anteroposterior and lateral diameters and gantry tilt were automatically measured by clicking the button "Calculate". 


\section{Results}

The resulting images depicting the diameters of AP and LAT for various gantry angles (from $0^{\circ}$ to $15^{\circ}$ ) are shown in Figure 6. At an angle of $0^{\circ}$, the AP and LAT diameters had the same length. The results of AP and LAT diameters and gantry angles from both manual and automatic measurements for various gantry angles and for two slice thicknesses (1.5 and $5 \mathrm{~mm}$ ) are shown in Table 1. Manual and automatic gantry angle calculations yielded the same results, and differences from the set angles for automatic calculation were less than $1^{\circ}$. The relationships between the results of automatic and manual gantry tilt calculations for slice thicknesses of 1.5 and $5 \mathrm{~mm}$ are shown in Figure 7. This shows linear relationships between them both with $\mathrm{R}^{2}>0.99$.
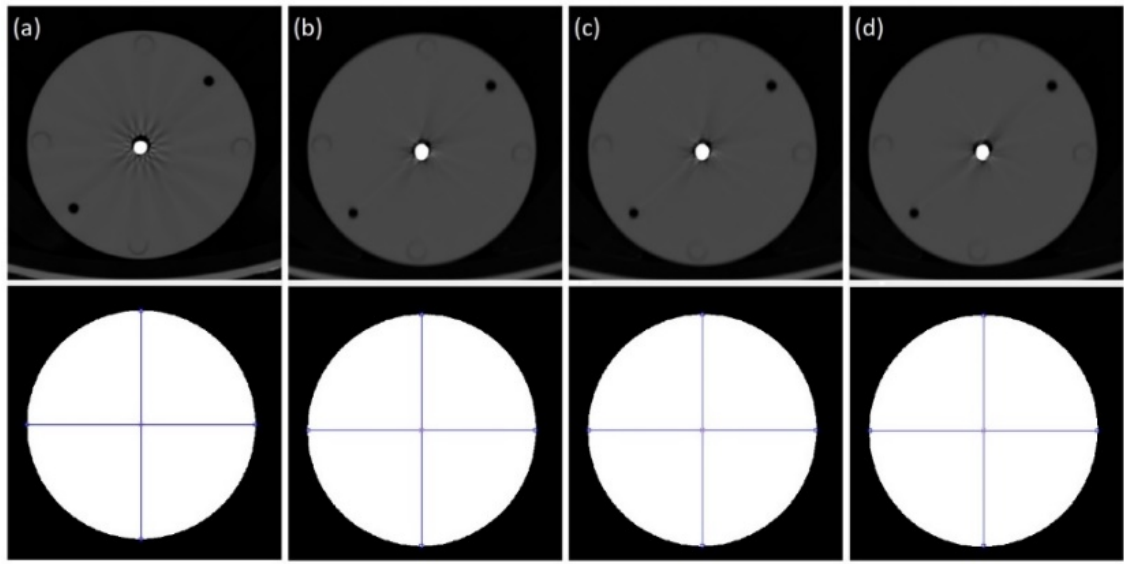

Figure 6. Images showing anteroposterior and lateral diameters from various gantry angles. (a) $0^{\circ}$, (b) $5^{\circ}$, (c) $10^{\circ}$, and (d) $15^{\circ}$.

Table 1. Results of anteroposterior and lateral diameters and gantry angles from both manual and automatic measurements for various gantry angles and two slice thicknesses.

\begin{tabular}{|c|c|c|c|c|c|}
\hline \multirow{2}{*}{$\begin{array}{c}\text { Set gantry angle } \\
\text { (degree) }\end{array}$} & \multirow{2}{*}{ Measured parameters } & \multicolumn{2}{|c|}{ Automatic } & \multicolumn{2}{|c|}{ Manual } \\
\hline & & Slice thickness of $1.5 \mathrm{~mm}$ & Slice thickness of $5 \mathrm{~mm}$ & Slice thickness of $1.5 \mathrm{~mm}$ & Slice thickness of $5 \mathrm{~mm}$ \\
\hline \multirow{3}{*}{0} & $\mathrm{AP}(\mathrm{mm})$ & $159.8 \pm 0.1$ & $159.8 \pm 0.1$ & $158.1 \pm 0.4$ & $158.4 \pm 0.2$ \\
\hline & LAT (mm) & $159.8 \pm 0.1$ & $159.8 \pm 0.2$ & $158.1 \pm 0.4$ & $158.4 \pm 0.2$ \\
\hline & Measured angle (degree) & $0.0 \pm 2.5$ & $0.0 \pm 1.8$ & $0.0 \pm 1.0$ & $0.0 \pm 1.1$ \\
\hline \multirow{3}{*}{5} & $\mathrm{AP}(\mathrm{mm})$ & $160.5 \pm 0.1$ & $160.4 \pm 0.2$ & $159.2 \pm 0.2$ & $158.9 \pm 0.3$ \\
\hline & LAT (mm) & $159.7 \pm 0.1$ & $159.6 \pm 1.0$ & $158.4 \pm 0.2$ & $158.1 \pm 0.2$ \\
\hline & Measured angle (degree) & $5.7 \pm 0.7$ & $5.6 \pm 1.0$ & $5.7 \pm 0.8$ & $5.5 \pm 0.3$ \\
\hline \multirow{3}{*}{10} & $\mathrm{AP}(\mathrm{mm})$ & $162.4 \pm 0.6$ & $162.3 \pm 0.3$ & $160.6 \pm 0.4$ & $160.5 \pm 0.3$ \\
\hline & $\operatorname{LAT}(\mathrm{mm})$ & $159.6 \pm 0.2$ & $159.6 \pm 0.2$ & $158.0 \pm 0.3$ & $157.9 \pm 0.2$ \\
\hline & Measured angle (degree) & $10.6 \pm 0.6$ & $10.5 \pm 0.7$ & $10.2 \pm 0.5$ & $10.2 \pm 0.5$ \\
\hline \multirow{3}{*}{15} & $\mathrm{AP}(\mathrm{mm})$ & $163.3 \pm 0.3$ & $162.3 \pm 0.4$ & $163.7 \pm 0.5$ & $163.1 \pm 0.4$ \\
\hline & $\operatorname{LAT}(\mathrm{mm})$ & $159.9 \pm 0.2$ & $159.9 \pm 0.2$ & $158.4 \pm 0.4$ & $157.9 \pm 0.3$ \\
\hline & Measured angle (degree) & $14.6 \pm 0.5$ & $14.6 \pm 0.6$ & $15.5 \pm 0.7$ & $14.4 \pm 0.6$ \\
\hline
\end{tabular}
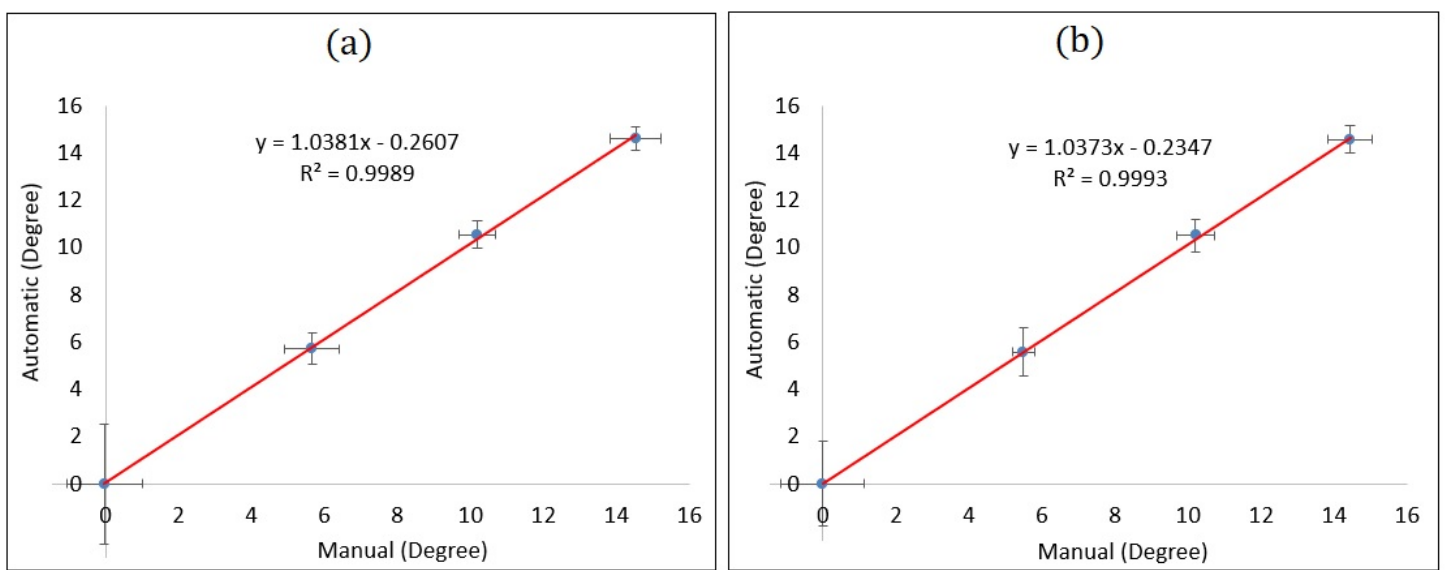

Figure 7. Relationships between the results of manual gantry tilt angle calculation and automatic gantry tilt angle calculation (degree) at slice thicknesses of $1.5 \mathrm{~mm}(\mathrm{a})$ and $5 \mathrm{~mm}(\mathrm{~b})$. 


\section{Discussion}

This study was aimed at implementing an effective method to automatically evaluate the accuracy of the CT gantry tilt calculation. The CT gantry tilt is generally performed, for example, in clinical head procedures to protect the eye lens from primary radiation if it is not the organ of interest. ${ }^{22}$ The gantry tilt is evaluated to ensure that the displayed angle in the gantry or console accurately indicates the true gantry tilt within a standard level legislated by the IAEA. ${ }^{23}$

The previous methods for evaluating the CT gantry tilt used a CR plate or film and required two scans of a cylindrical water phantom. ${ }^{16}$ The current method of evaluating the accuracy of the gantry tilt is more straightforward because it only uses one scan using a readily available phantom, i.e. head PMMA phantom. The evaluation of the CT gantry tilt is easy, fast, and convenient.

We only used angles between $0^{\circ}$ and $15^{\circ}$, corresponding to the maximum angle used in clinical settings at our hospital. A further study should be performed to investigate the accuracy of the proposed method with angles of up to $30^{\circ}$ or the maximum tilt angle of the CT.

Slice thicknesses $(1.5$ and $5 \mathrm{~mm})$ were also limited. However, these limited thicknesses are sufficient as proof of concept. Theoretically, the proposed method is influenced by slice thickness. At the same gantry angle, a wider slice thickness gives a larger gantry tilt measurement. In clinical practice, the narrowest slice thickness should be chosen for a more accurate result.

In this study, the phantom used was a head PMMA phantom with a diameter of $16 \mathrm{~cm}$. However, other phantoms, for example, a body PMMA phantom with a diameter of $32 \mathrm{~cm}$, or any cylindrical phantom, such as cylindrical water or Catphan or ACR phantom, can be used for automatic CT gantry evaluation. The important thing is that in the proposed method, the phantom must be correctly positioned in the horizontal axis. If the phantom is slightly tilted horizontally, it will reduce the accuracy of the gantry tilt calculation because of changes in the LAT diameter.

The gantry tilt is used for not only head CT examinations but also other examinations, such as guiding examination procedures as a needle pointing device for tissue removal. ${ }^{22}$

\section{Conclusion}

An effective method for the CT gantry tilt evaluation using a single scan of an easily available cylindrical phantom has been proposed. Software has been developed to determine it automatically. The method is accurate and precise with an error within $1^{\circ}$. The automatic method yields the same results as the manual method. This approach offers a convenient method for CT gantry tilt evaluation, which could be used for regular quality control.

\section{References}

1. Kalender WA. X-ray computed tomography. Phys Med Biol. 2006;51:29-43. https://doi.org/10.1088/0031-9155/51/13/R03

2. Seeram, E. Computed tomography: Physical principles, clinical applications, and quality control. Elsevier. Fourth edition. 2016.

3. Ebrahiminia A, Asadinezhad M, Mohammadi F, Khoshgard K. Eye lens dose optimization through gantry tilting in brain ct scan: the potential effect of the radiological technologists' training. Radiat Prot Dosimetry. 2020;189:527-33. https://doi.org/10.1093/rpd/ncaa073

4. Anam C, Fujibuchi T, Haryanto F, et al. An evaluation of computed tomography dose index measurements using a pencil ionisation chamber and small detectors. J Radiol Prot. 2019;39:112-24. https://doi.org/10.1088/1361-6498/aaf2b4

5. Anam C, Fujibuchi T, Toyoda T. et al. The impact of head miscentering on the eye lens dose in CT scanning: Phantoms study. J Phys Conf Ser. 2019;1204:012022. https://doi.org/10.1088/1742-6596/1204/1/012022

6. International Commission on Radiological Protection. Annals of the ICRP Annals of the ICRP Annals of the ICRP. ICRP Publication 92, annals of ICRP 28. 2003.

7. Ishizaka H, Naka M, Nagase H, et al. A new brain CT reference line:the lower eyelid to the inner occipital base line closely parallels the Talairach-Tournoux line. Acta Radiologica Open. 2020;9:1-6. https://doi.org/10.1177/2058460120902406

8. Nishizawa K, Maruyama T, Takayama M, Okada M, Hachiya J, Furuya Y. Determinations of organ doses and effective dose equivalents from computed tomographic examination. Br J Radiol. 1991;64:20-8. https://doi.org/10.1259/0007-1285-64-757-20

9. Maclennan AC. Radiation dose to the lens from CT brain scans in general radiology departments. Br J Radiol. 1995;68:219. https://doi.org/10.1259/0007-1285-68-806-219

10. Poon R, Badawy MK. Radiation dose and risk to the lens of the eye during CT examinations of the brain. J Med Imaging Radiat Oncol. 2019;63:786-94. https://doi.org/10.1111/1754-9485.12950

11. Nikupaavo U, Kaasalainen T, Reijonen V, Ahonen SM, Kortesniemi M. Lens dose in routine head CT: Comparison of different optimization methods with anthropomorphic phantoms. Am J Roentgenol. 2015;204:117-23. https://doi.org/10.2214/AJR.14.12763

12. Fung KKL, Choi KHW, Hom H. Lens dose reduction in paediatric ct brain scan using the supra-orbitomeatal baseline technique. ECR 2005. 2005. https://doi.org/10.1594/ECR05/C-0994 
13. Parsi M, Sohrabi M, Mianji F, Paydar R. Gantry angulation effects on CT dose along the z-axis direction in head examinations. Radiat Prot Dosimetry. 2017;177:458-65. https://doi.org/10.1093/rpd/ncx064

14. Ali STM, Hamad MM, Ayad CE, Abdalla EA, Ahmed AS. Evaluation of the technical specifications of computerized tomography scanners in Jazan. Sudan Med Monit. 2013;8:159-66. https://doi.org/10.4103/1858-5000.132611

15 Abou-Elenein HS. Quality assurance for computed-tomography simulator: In home Z-phantom for mechanical tests of the couch and the gantry. Chinese-German J Clin Oncol. 2013;12(5):237-242. 2013. https://doi.org/10.1007/s10330-012-1133-3

16. Diagnostic X-Ray Imaging Committee. Specification and Acceptance Testing of Computed Tomography Scanners. AAPM Report No. 39. New York: the American Institute of Physics, Inc. 1993.

17. Sharma DS, Sharma SD, Sanu KK, Saju S, Deshpande DD, Kannan S. Performance evaluation of a dedicated computed tomography scanner used for virtual simulation using in-house fabricated CT phantoms. J Med Phys. 2006;31:28-35. https://doi.org/10.4103/0971-6203.25667

18. American Association of Physicists in Medicine. Specification and acceptance testing of computed tomography scanners. AAPM Report No. 39. 1993.

19. Anam C, Haryanto F, Widita R, Arif I. Automated Estimation of Patient's Size from 3D Image of Patient for Size Specific Dose Estimates (SSDE). Adv Sci Eng Med. 2015;7:892-6. https://doi.org/10.1166/asem.2015.1780

20. Anam C. Haryanto F, Widita R, Arif I, Dougherty G. A fully automated calculation of size-specific dose estimates (SSDE) in thoracic and head CT examinations. J Phys Conf Ser. 2016;694:012030. https://doi.org/10.1088/1742-6596/694/1/012030

21. Anam C, Haryanto F, Widita R, Arif I, Dougherty G. Automated calculation of water-equivalent diameter (DW) based on AAPM task group 220. J Appl Clin Med Phys. 2016;17:32033. https://doi.org/10.1120/jacmp.v17i4.6171

22. Boulter DJ, Rumboldt Z, Bonaldi G, Muto M, Cianfoni A. Tilting the gantry for CT-guided spine procedures. Radiol Medica. 2014;119:750-7. https://doi.org/10.1007/s11547-013-0344-1

23. IAEA. Quality assurance programme for computed tomography: Diagnostic and therapy applications. IAEA Huma. Health Series No. 19. 2012. 